\title{
Features and treatment modality of iliopsoas abscess and its outcome: a 6-year hospital-based study
}

Ming-Shun Hsieh ${ }^{1,2,3}$, Shih-Che Huang ${ }^{1}$, El-Wui Loh ${ }^{4}$, Che-An Tsai ${ }^{5}$, Ying-Ying Hung ${ }^{6}$, Yu-Tse Tsan ${ }^{1,2}$, Jin-An Huang ${ }^{1,7}$, Lee-Min Wang ${ }^{1,8,9}$ and Sung-Yuan $\mathrm{Hu}^{1,2,3,10^{*}}$

\begin{abstract}
Background: Percutaneous drainage (PCD) and surgical intervention are two primary treatment options for iliopsoas abscess (IPA). However, there is currently no consensus on when to use PCD or surgical intervention, especially in patients with gas-forming IPA. This study compared the characteristics of patients with gas-forming and non-gas forming IPA and their mortality rates under different treatment modalities. An algorithm for selecting appropriate treatment for IPA patients is proposed based on our findings.
\end{abstract}

Methods: Eighty-eight IPA patients between July 2007 and February 2013 were enrolled in this retrospective study. Patients $<18$ years of age or with an incomplete course of treatment were excluded. Demographic information, clinical characteristics, and outcomes of different treatment approaches were compared between gas-forming IPA and non-gas forming IPA patients.

Results: Among the 88 enrolled patients, 27 (31\%) had gas-forming IPA and 61 (69\%) had non-gas forming IPA. The overall intra-hospital mortality rate was $25 \%$. The gas-forming IPA group had a higher intra-hospital mortality rate $(12 / 27,44.0 \%)$ than the non-gas forming IPA group $(10 / 61,16.4 \%)(P<0.001)$. Only 2 of the 13 patients in the gas-forming IPA group initially accepting PCD had a good outcome (success rate $=15.4 \%$ ). Three of the 11 IPA patients with failed initial PCD expired, and 8 of the 11 patients with failed initial PCD accepted salvage operation, of whom 5 survived. Seven of the 8 gas-forming IPA patients accepting primary surgical intervention survived (success rate $=87.5 \%$ ). Only 1 of the 6 gas-forming IPA patients who accepted antibiotics alone, without PCD or surgical intervention, survived (success rate $=16.7 \%$ ). In the non-gas forming IPA group, 23 of 61 patients initially accepted $P C D$, which was successful in 17 patients (73.9\%). The success rate of PCD was much higher in the non-gas forming group than in the gas-forming group $(P<0.01)$.

Conclusions: Based on the high failure rate of PCD and the high success rate of surgical intervention in our samples, we recommend early surgical intervention with appropriate antibiotic treatment for the patients with gas-forming IPA. Either PCD or primary surgical intervention is a suitable treatment for patients with non-gas forming IPA.

Keywords: Iliopsoas abscess, Percutaneous drainage, Psoas abscess, Pyomyositis, Surgery

\footnotetext{
* Correspondence: song9168@pie.com.tw

'Department of Emergency Medicine, Taichung Veterans General Hospital, Taichung, Taiwan

${ }^{2}$ School of Medicine, Chung Shan Medical University, Taichung, Taiwan

Full list of author information is available at the end of the article
} 


\section{Background}

Iliopsoas abscess (IPA), a suppurative collection within the compartment of the psoas and iliacus muscles, was first reported by Mynter et al. in 1881 [1]. It is an infrequent but potentially life-threatening infectious disease. Prior to introduction of effective anti-tuberculosis drugs, IPA was once a common complication of tuberculous spinal infection. With the common application of anti-tuberculosis drugs, non-tuberculous pyogenic IPA has become the predominant form. Clinically, IPA is classified according to its origin [2]. Primary IPA is thought to be secondary to an unrecognized staphylococcal bacteremia, while secondary IPA is caused by underlying conditions such as gastrointestinal or genitourinary tract diseases, spread of infection from postoperative aortic aneurysm, spondylodiskitis, osteomyelitis, septic arthritis, and infection subsequent to renal surgery $[1,3]$. Primary IPA is often seen in younger patients and in developing or tropical countries, while secondary IPA occurs more frequently in developed countries with mixed enteric flora [2]. In recent decades, the incidence of primary IPA has gradually increased as numbers of intravenous drug-abusers and those afflicted with human immunodeficiency virus (HIV) infection increase [1,4].

The psoas-muscle sign, the triad of fever, flank pain, and limitation of hip movement, is noted in only $30 \%$ patients [5]. Because of the rapid progress in advanced imaging techniques, such as gallium-67 scanning, computed tomography (CT), and magnetic resonance image (MRI) in recent years, early diagnosis of IPA has become easier despite initial clinical presentation that is frequently ambiguous [6-8]. Although origin-based IPA classification has been adopted in clinical practice, it does not help clinicians make appropriate treatment decisions. Currently, whether percutaneous drainage (PCD) or surgical intervention should be used in IPA patients remains controversial because few studies with large sample sizes have been performed. At present, PCD is considered preferable to surgical intervention for the treatment of IPA [9-11].

In this study, we compared demographic information, laboratory data, microbiological distribution, origin of infection, treatment modality (i.e., antibiotics alone, PCD, and surgical intervention), and clinical outcomes between patients with gas-forming IPA and non-gas forming IPA. From these results, we established an evidencebased algorithm for selecting an appropriate treatment modality for IPA.

\section{Methods}

This retrospective study was approved by the institutional review board of Taichung Veterans General Hospital (No. CE13129). The data were retrieved from the electronic clinical database of Taichung Veterans General Hospital, a 1,520-bed referral medical center in central Taiwan. The diagnosis of IPA was confirmed by (1) documentation of abscess within the iliopsoas muscle by either PCD or surgery; or (2) classic CT or MRI finding of IPA with compatible clinical presentation, laboratory data, and blood cultures. Typical CT or MRI images in IPA patients included the following features: (1) enlargement of affected muscle, (2) rim enhancement of the abscess wall with lower central attenuation, and (3) gas presence either as an air-fluid interface or mottled air bubbles. All CT and MRI images were reviewed by the primary study investigators and one radiologist.

The primary outcome of this study was the intrahospital mortality of treated IPA patients. We defined success of treatment as improvement in clinical condition, decrement of IPA size in follow-up images, and discharge alive after treatment with $\mathrm{PCD}$, surgery, or antibiotics alone. Failure of treatment was defined as mortality during hospitalization or deterioration of a patient's clinical condition with accompanying non-decrement of IPA size on follow-up images necessitating another advanced treatment modality (i.e., antibiotics alone plus PCD, antibiotics alone plus surgery, or PCD followed by surgery). All the patients accepted a minimum follow-up period of 2 months. Cases were excluded if the patient was $<18$ years old or had an incomplete treatment course.

Eighty-eight IPA patients admitted to the hospital between July 2007 and February 2013 were included in this study. Demographic data, laboratory studies, etiological pathogens, infection origins, management approaches, clinical courses, and patient's outcomes were coded for further statistical analysis. Continuous variables were reported as mean $\pm \mathrm{SD}$, whereas categorical variables were described as the number and percentage of subjects. Patients were divided into gas-forming IPA and non-gas forming IPA groups. Further comparisons were performed using Mann-Whitney $U$ test for continuous variables and chi-square test or Fisher's exact test for categorical variables. A $P$ value of less than 0.05 was accepted as statistically significant. Statistical analysis was performed using the Statistical Package for the Social Sciences Version 15.1 (SPSS Inc., Chicago, IL, USA).

\section{Results}

\section{Clinical features and biochemistry}

Of the 88 IPA patients enrolled (58 females, 30 males; mean age $63.0 \pm 15.6$ years [range, 29-93 years]), 29 patients (33.0\%) had bilateral IPA and $74(84.1 \%)$ had multiloculated IPA determined on CT. Mean maximal transverse IPA cavity diameter on CT was $3.98 \pm 2.31 \mathrm{~cm}$. Fever or hypothermia was noted in 74 patients $(84.0 \%)$ upon initial presentation to the hospital. IPA was of primary origin in 21 patients (24\%) and of secondary 
origin in 67 (76\%). Severe sepsis was noted in 53 patients (60.2\%) upon initial presentation. The average length of hospital stay was $36 \pm 39$ days (range, 2-328 days; median, 28 days) and the intra-hospital mortality rate was $25 \%$.

\section{Etiological pathogens}

The microbiological documentation rate of all IPA patients via either pus or correlated blood cultures was 83.0\% (73/88), including 59.3\% (51/86) with positive blood culture, and $75.7 \%(53 / 70)$ with positive pus culture. Staphylococcus spp. were the most common pathogens in this study, found in blood cultures in 27 patients and pus cultures in 26; followed by Streptococcus spp.; Klebsiella pneumoniae; and Escherichia coli. Methicillin-resistant Staphylococcus aureus (MRSA) was found in blood cultures in 10 patients and pus cultures in 9 patients. Anaerobic blood cultures were positive in 7 patients and anaerobic pus cultures were positive in 13 patients. Only one pus culture was positive for tuberculosis.

\section{Origins of secondary IPA}

Among the 61 secondary IPA patients, the most common etiologies were skeletal (37 patients, 60.7\%), followed by cardiovascular (11 patients, $18.0 \%$ ) and urinary tract (10 patients, 16.4\%) (Table 1). Cardiovascular origins of IPA included 3 cases (4.9\%) of infective endocarditis with distal septic infection, 4 cases $(6.6 \%)$ of abdominal aortic aneurysm with subsequent infection after endovascular stent implantation, and 2 cases (3.3\%) of infected aortic aneurysm.

\section{Imaging studies}

CT confirmed the diagnosis of IPA in all patients. MRI was performed in 20 patients with poor treatment response or high suspicion for infection of adjacent structures. Accordingly, osteomyelitis/spondylodiskitis and epidural abscess were demonstrated in 18 (20.5\%) and 11 (12.5\%) patients, respectively. The 11 patients with epidural abscess underwent subsequent surgical intervention and 3 expired.

\section{Causes of mortality}

The ultimate causes of mortality of our patients were examined by two research physicians via review of medical records. In controversial cases, the primary care physicians were consulted and consensus was reached after thorough discussion. There were 22 mortality cases in our study group, and 16 cases (72.7\%) expired due to refractory sepsis. Six cases (27.3\%) expired because of comorbidities, including 2 from massive upper gastrointestinal bleeding and 2 from hemorrhagic shock caused by aortic aneurysm rupture. The causes of mortality of the remaining 2 cases were unexpected cardiac arrest and end-stage cancer.
Table 1 Classification of IPA origin between gas-forming IPA and non-gas forming IPA patients

\begin{tabular}{|c|c|c|}
\hline & $\begin{array}{l}\text { Gas-forming } \\
\text { IPA }(n=27)\end{array}$ & $\begin{array}{l}\text { Non-gas forming } \\
\text { IPA }(n=61)\end{array}$ \\
\hline Origin (Primary:Secondary) & $3: 24$ & $18: 43$ \\
\hline Skeletal & $11(40.7 \%)$ & $26(42.6 \%)$ \\
\hline $\begin{array}{l}\text { Vertebral osteomyelitis/ } \\
\text { spondylodiskitis }\end{array}$ & 10 & 17 \\
\hline Ilio-sacral joint septic arthritis & 0 & 7 \\
\hline Hip septic arthritis & 1 & 2 \\
\hline Intra-abdominal & $1(3.7 \%)$ & $4(6.6 \%)$ \\
\hline Necrotizing pancreatitis & 0 & 2 \\
\hline Appendicitis & 0 & 1 \\
\hline Colon cancer with abscess & 1 & 0 \\
\hline Post Gl procedure & 0 & $1^{a}$ \\
\hline Cardiovascular & $6(22.2 \%)$ & $5(8.2 \%)$ \\
\hline $\begin{array}{l}\text { Abdominal aortic aneurysm post- } \\
\text { stent implantation }\end{array}$ & 2 & 2 \\
\hline Infected aortic aneurysm & 2 & 0 \\
\hline Infective endocarditis & 0 & 3 \\
\hline Catheter related & 2 & 0 \\
\hline Urinary tract & $5(18.5 \%)$ & $5(8.2 \%)$ \\
\hline Acute pyelonephritis & $2^{b}$ & $2^{c}$ \\
\hline Post GU procedure & $3^{d}$ & $2^{e}$ \\
\hline Prostate abscess & 0 & 1 \\
\hline Post gynecologic procedure & 0 & $1^{f}(1.6 \%)$ \\
\hline $\begin{array}{l}\text { Empyema with downward } \\
\text { extension }\end{array}$ & 0 & $2(3.3 \%)$ \\
\hline Limb necrotizing fasciitis & $1(3.7 \%)$ & 0 \\
\hline
\end{tabular}

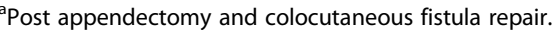

bIncluding 2 EPN

c Including 1 EPN.

dIncluding 1 post nephrectomy, 1 post Bench surgery, and 1 renal stone post ESWL.

Including 2 post nephrectomy.

Endometrioid cancer post operation.

EPN, emphysematous pyelonephritis; ESWL, extracorporeal shock wave lithotripsy; Gl, gastrointestinal; GU, genitourinary.

\section{Initial treatment options}

Once the patients with suspicious infection presented to the hospital, empirical antibiotics were prescribed within 2 hours. Third-generation cephalosporin plus metronidazole $(15 / 88,17.0 \%)$ or carbapenem $(13 / 88,14.8 \%)$ were most often prescribed initially to our study cases. Antibiotics were adjusted after demonstration of IPA via advanced image studies or available blood/pus cultures. PCD and surgery were the standard treatment modalities for IPA if drainage was indicated. Patients who did not require (because the condition was mild) or were not candidates for (because the condition was too severe for invasive treatment) PCD or surgery received therapy with antibiotics alone. 
A comparison of characteristics of IPA patients between different initial treatment modalities (antibiotics alone, PCD, or surgery) is shown in Table 2. Patients who underwent antibiotic therapy alone were older $(P=0.005)$ and had smaller mean diameter of the IPA cavity $(P<0.0001)$ and lower platelet count $(P=0.016)$ than patients receiving other forms of treatment. Impaired renal function was more frequent in this group $(P<0.05)$. Shorter length of hospital stay $(P=0.001)$ and higher mortality rate $(P=$ 0.002 ) were also observed. Other variables were not different between the three groups. Initial treatment with antibiotics alone was chosen in our study group when the IPA was too small to be drained by PCD $(n=9)$ or when patients were too weak to undergo an invasive procedure $(\mathrm{n}=15)$.

\section{Factors contributing to mortality}

Possible contributions of age, sex, clinical and laboratory variables to mortality were analyzed using univariate analysis (Table 3). Results revealed that age, gas-forming IPA, white blood cell (WBC) count, platelet count, blood urea nitrogen (BUN), creatinine, potassium, and secondary IPA origin were significantly associated with mortality. Further Cox regression analysis of these variables as well as bilateral IPA revealed that none was associated with mortality rate except initial treatment. The hazard ratio (HR) for treatment using antibiotics alone was 5.34 (95\% confidence interval [CI], 1.27-22.56), whereas surgery had no effect on mortality rate $(\mathrm{HR}=0.28,95 \% \mathrm{CI}$ 0.04-2.02, $P=0.207$ )

Table 2 Comparison of patient characteristics between initial treatment with antibiotics alone, PCD, or surgery

\begin{tabular}{|c|c|c|c|c|}
\hline & $\begin{array}{c}\text { Antibiotics }(n=24) \\
\text { Mean } \pm S D\end{array}$ & $\begin{array}{c}\text { PCD }(n=36) \\
\text { Mean } \pm S D\end{array}$ & $\begin{array}{c}\text { Surgery }(n=28) \\
\text { Mean } \pm \text { SD }\end{array}$ & $P$ value \\
\hline Age (years) & $72.0 \pm 13.8$ & $59.6 \pm 15.6$ & $60.0 \pm 14.6$ & $0.005^{*}$ \\
\hline $\operatorname{Sex}(F: M)$ & $15: 9$ & $23: 13$ & $20: 8$ & 0.752 \\
\hline Gas-forming IPA & $18(75.0 \%)$ & $23(63.9 \%)$ & $20(71.4 \%)$ & 0.631 \\
\hline Bilateral & $10(41.7 \%)$ & $10(27.8 \%)$ & $9(32.1 \%)$ & 0.530 \\
\hline Multiloculated IPA collection pattern & $17(70.8 \%)$ & $33(91.7 \%)$ & $24(85.7 \%)$ & 0.093 \\
\hline Mean maximal transverse IPA cavity diameter on $C T(\mathrm{~cm})$ & $2.2 \pm 1.5$ & $5.2 \pm 2.1$ & $4.0 \pm 2.2$ & $<0.0001^{*}$ \\
\hline $\mathrm{DM}$ & $10(41.7 \%)$ & $13(36.1 \%)$ & $11(39.3 \%)$ & 0.907 \\
\hline Hypertension & $8(33.3 \%)$ & $12(33.3 \%)$ & $13(46.4 \%)$ & 0.497 \\
\hline CKD & $9(37.5 \%)$ & $7(19.4 \%)$ & $3(10.7 \%)$ & 0.060 \\
\hline Malignancy & $4(16.7 \%)$ & $9(25.0 \%)$ & $4(14.3 \%)$ & 0.520 \\
\hline Liver cirrhosis & $4(16.7 \%)$ & $6(16.7 \%)$ & $2(7.1 \%)$ & 0.479 \\
\hline Fever or hypothermia & $20(83.3 \%)$ & $31(86.1 \%)$ & $23(82.1 \%)$ & 0.905 \\
\hline Severe sepsis & $18(75.0 \%)$ & $29(80.6 \%)$ & $21(75.0 \%)$ & 0.830 \\
\hline WBC $(/ \mu \mathrm{L})$ & $18900 \pm 9428$ & $17672 \pm 9018$ & $15354 \pm 7426$ & 0.344 \\
\hline Bandemia (No. of patients) & $12(50.0 \%)$ & $17(47.2 \%)$ & $9(32.1 \%)$ & 0.353 \\
\hline $\mathrm{Hb}(\mathrm{g} / \mathrm{dL})$ & $10.8 \pm 2.4$ & $10.7 \pm 2.3$ & $10.7 \pm 2.7$ & 0.996 \\
\hline Platelet $\left(\times 10^{3} / \mu \mathrm{L}\right)$ & $185.5 \pm 140.9$ & $309.2 \pm 179.0$ & $255.6 \pm 144.8$ & $0.016^{*}$ \\
\hline BUN (mg/dL) & $65.6 \pm 67.1$ & $36.8 \pm 37.1$ & $32.4 \pm 28.0$ & $0.041^{*}$ \\
\hline Creatinine (mg/dL) & $3.5 \pm 4.0$ & $2.2 \pm 2.0$ & $1.43 \pm 0.9$ & $0.037^{*}$ \\
\hline $\operatorname{ALT}(U / L)(n=24$ vs 35 vs 28) & $106.3 \pm 250.6$ & $51.0 \pm 77.7$ & $80.1 \pm 244.9$ & 0.768 \\
\hline $\mathrm{Na}(\mathrm{mEq} / \mathrm{L})$ & $132.3 \pm 8.5$ & $133.4 \pm 5.6$ & $135.0 \pm 5.5$ & 0.258 \\
\hline $\mathrm{K}(\mathrm{mEq} / \mathrm{L})$ & $4.4 \pm 1.0$ & $4.0 \pm 0.5$ & $4.1 \pm 0.7$ & 0.223 \\
\hline $\mathrm{CRP}(\mathrm{mg} / \mathrm{L})$ & $16.0 \pm 10.5$ & $21.9 \pm 10.1$ & $19.3 \pm 12.2$ & 0.083 \\
\hline CK (U/L) (n= 22 vs 29 vs 25) & $539.3 \pm 1711.5$ & $175.6 \pm 302.3$ & $183.1 \pm 535.1$ & 0.309 \\
\hline Initial blood glucose (mg/dL) (n= 24 vs 34 vs 28) & $180.0 \pm 116.0$ & $208.0 \pm 151.3$ & $155.3 \pm 88.6$ & 0.369 \\
\hline Primary:Secondary origin & $8: 16$ & $8: 28$ & $5: 23$ & 0.408 \\
\hline Mortality & $12(50.0 \%)$ & $8(22.2 \%)$ & $2(7.1 \%)$ & $0.002^{*}$ \\
\hline Length of hospital stay (days) & $19.4 \pm 15.2$ & $44.9 \pm 55.3$ & $38.8 \pm 19.5$ & $0.001^{*}$ \\
\hline
\end{tabular}

$* P<0.05$.

ALT, alanine aminotransferase; BUN, blood urea nitrogen; $C K$, creatine kinase; CKD, chronic kidney disease; CRP, C-reactive protein; CT, computed tomography; DM, diabetes mellitus; $\mathrm{Hb}$, hemoglobin; IPA, iliopsoas abscess; $\mathrm{K}$, potassium; Na, sodium; PCD, percutaneous drainage; SD, standard deviation; WBC, white blood cells. 
Table 3 Univariate and multivariate analyses of effects of age, sex and clinical variables on mortality rate

\begin{tabular}{|c|c|c|c|c|c|c|}
\hline & & Jnivariate anal & & & ultivariate ana & \\
\hline & HR & $95 \% \mathrm{Cl}$ & $P$ value & HR & $95 \% \mathrm{Cl}$ & $P$ value \\
\hline Age (years) & 1.05 & $(1.010-1.083)$ & $0.013^{*}$ & 1.03 & $(0.986-1.071)$ & 0.204 \\
\hline Sex-female & 1.06 & $(0.421-2.655)$ & 0.905 & 1.21 & $(0.369-3.997)$ & 0.749 \\
\hline Gas-forming IPA & 2.57 & $(1.060-6.250)$ & $0.037^{*}$ & 1.93 & $(0.425-8.804)$ & 0.393 \\
\hline Bilateral IPA & 2.28 & $(0.943-5.524)$ & 0.067 & 1.76 & $(0.563-5.481)$ & 0.332 \\
\hline Multiloculated IPA collection pattern & 0.85 & $(0.248-2.938)$ & 0.801 & & & \\
\hline Mean maximal transverse IPA cavity diameter on $\mathrm{CT}(\mathrm{cm})$ & 0.93 & $(0.747-1.162)$ & 0.533 & & & \\
\hline DM & 1.21 & $(0.490-2.996)$ & 0.679 & & & \\
\hline Hypertension & 0.41 & $(0.145-1.134)$ & 0.085 & & & \\
\hline CKD & 1.22 & $(0.459-3.265)$ & 0.687 & & & \\
\hline Malignancy & 1.34 & $(0.442-4.057)$ & 0.605 & & & \\
\hline Liver cirrhosis & 1.19 & $(0.346-4.080)$ & 0.785 & & & \\
\hline Fever or hypothermia & 1.53 & $(0.351-6.647)$ & 0.572 & & & \\
\hline Severe sepsis & 29.17 & $(0.215-3952)$ & 0.178 & & & \\
\hline WBC $(/ \mu \mathrm{L})$ & 1.00 & $(1.000-1.000)$ & $0.004^{*}$ & 1.00 & $(1.000-1.000)$ & 0.109 \\
\hline Bandemia & 1.76 & $(0.722-4.315)$ & 0.213 & & & \\
\hline $\mathrm{Hb}(\mathrm{g} / \mathrm{dL})$ & 0.89 & $(0.737-1.084)$ & 0.255 & & & \\
\hline Platelet $\left(\times 10^{3} / \mu \mathrm{L}\right)$ & 1.00 & (0.992-0.999) & $0.013^{*}$ & 1.00 & $(0.992-1.001)$ & 0.109 \\
\hline $\mathrm{BUN}(\mathrm{mg} / \mathrm{dL})$ & 1.01 & $(1.005-1.017)$ & $0.001^{*}$ & 1.00 & $(0.983-1.010)$ & 0.623 \\
\hline Creatinine $(\mathrm{mg} / \mathrm{dL})$ & 1.12 & $(1.009-1.233)$ & $0.033^{*}$ & 0.93 & $(0.738-1.178)$ & 0.555 \\
\hline $\mathrm{ALT}(\mathrm{U} / \mathrm{L})$ & 1.00 & $(0.998-1.002)$ & 0.702 & & & \\
\hline $\mathrm{Na}(\mathrm{mEq} / \mathrm{L})$ & 1.01 & $(0.946-1.083)$ & 0.721 & & & \\
\hline $\mathrm{K}(\mathrm{mEq} / \mathrm{L})$ & 2.54 & $(1.442-4.485)$ & $0.001^{*}$ & 1.36 & $(0.674-2.761)$ & 0.389 \\
\hline $\mathrm{CRP}(\mathrm{mg} / \mathrm{L})$ & 0.98 & $(0.935-1.021)$ & 0.303 & & & \\
\hline CK (U/L) & 1.00 & $(0.999-1.001)$ & 0.972 & & & \\
\hline Initial blood glucose (mg/dL) & 1.00 & $(0.996-1.003)$ & 0.750 & & & \\
\hline Secondary IPA origin & 0.37 & $(0.149-0.931)$ & $0.035^{*}$ & 0.35 & $(0.081-1.490)$ & 0.154 \\
\hline Initial treatment & & & & & & \\
\hline Antibiotics alone & 6.49 & $(2.132-19.753)$ & $0.001^{*}$ & 5.34 & $(1.266-22.558)$ & $0.023^{*}$ \\
\hline PCD & & erence & & & ference & \\
\hline Surgery & 0.51 & $(0.098-2.627)$ & 0.418 & 0.28 & $(0.039-2.017)$ & 0.207 \\
\hline
\end{tabular}

adjusted for age, gender, gas-forming IPA, bilateral IPA, WBC, Platelet, BUN, creatinine, K, secondary IPA origin, and initial treatment.

${ }^{*} \mathrm{P}<0.05$.

$\mathrm{ALT}$, alanine aminotransferase; BUN, blood urea nitrogen; $\mathrm{CK}$, creatine kinase; $\mathrm{CKD}$, chronic kidney disease; CRP, C-reactive protein; CT, computed tomography; DM, diabetes mellitus; Hb, hemoglobin; HR, hazard ratio; IPA, iliopsoas abscess; K, potassium; Na, sodium; PCD, percutaneous drainage; SD, standard deviation; WBC, white blood cells.

\section{Comparison between patients with gas-forming and non-gas forming IPA}

Results of comparisons between gas-forming IPA and non-gas forming IPA patients are summarized in Table 4. Among the 88 patients enrolled, 27 (30.7\%) had gasforming IPA and 61 (69.3\%) had non-gas forming IPA. No sex predominance between groups was identified. Patients in the gas-forming IPA group showed a higher incidence of bilateral involvement $(14 / 27,51.9 \%)$ than the non-gas forming IPA group $(15 / 61,24.6 \%)(P<0.05)$. Neither the incidence of multiloculated IPA collection pattern (92.5\% versus $80.3 \%)$ nor the mean maximal transverse IPA cavity diameter $(4.21 \mathrm{~cm}$ versus $3.87 \mathrm{~cm}$ ) was significantly different between the 2 groups. The gas-forming IPA group had a higher intra-hospital mortality rate $(12 / 27,44.0 \%)$ than the non-gas forming IPA group $(10 / 61,16.4 \%)(P<0.001)$. No difference in underlying disease, primary versus secondary origin, biochemistry profile, or average length of hospital stay was found between the 2 groups.

The overall microbiological documentation rate was $85.2 \%(23 / 27)$ in patients with gas-forming IPA and 
Table 4 Comparison between gas-forming IPA and non-gas forming IPA patients

\begin{tabular}{|c|c|c|c|}
\hline & $\begin{array}{c}\text { Gas-forming } \\
\text { IPA } \\
(\mathrm{n}=27) \\
\text { Mean } \pm \text { SD }\end{array}$ & $\begin{array}{c}\text { Non-gas } \\
\text { forming IPA } \\
(n=61) \\
\text { Mean } \pm \text { SD }\end{array}$ & $P$ value \\
\hline Age (years) & $67.8 \pm 13.9$ & $61.1 \pm 16.0$ & 0.053 \\
\hline Sex (F:M) & $14: 13$ & $44: 17$ & 0.064 \\
\hline Bilateral & $14(51.9 \%)$ & $15(24.6 \%)$ & $0.012^{*}$ \\
\hline $\begin{array}{l}\text { Multiloculated IPA } \\
\text { collection pattern }^{f}\end{array}$ & 25(92.6\%) & 49(80.3\%) & 0.211 \\
\hline $\begin{array}{l}\text { Mean maximal transverse IPA } \\
\text { cavity diameter on } \mathrm{CT}(\mathrm{cm})\end{array}$ & $4.21 \pm 1.79$ & $3.87 \pm 2.51$ & 0.166 \\
\hline $\mathrm{DM}$ & 13(48.2\%) & $21(34.4 \%)$ & 0.223 \\
\hline Hypertension & $8(29.6 \%)$ & $25(41.7 \%)$ & 0.284 \\
\hline Uremia $^{f}$ & $3(11.1 \%)$ & $8(13.1 \%)$ & 1.000 \\
\hline CKD & $5(18.5 \%)$ & $14(23.0 \%)$ & 0.641 \\
\hline Malignancy & $4(14.8 \%)$ & 13(21.7\%) & 0.456 \\
\hline Liver cirrhosis ${ }^{f}$ & $1(3.7 \%)$ & $11(18.0 \%)$ & 0.096 \\
\hline \multirow[t]{2}{*}{ Fever or hypothermia ${ }^{f}$} & $26(96.3 \%)$ & $48(78.7 \%)$ & 0.056 \\
\hline & $\begin{array}{l}\text { (Including } 2 \\
\text { hypothermia) }\end{array}$ & $\begin{array}{l}\text { (Including } 4 \\
\text { hypothermia) }\end{array}$ & \\
\hline Severe sepsis & $27(100.0 \%)$ & $26(67.2 \%)$ & $0.002^{*}$ \\
\hline WBC $(/ \mu \mathrm{L})$ & $20363 \pm 11077$ & $15900 \pm 7059$ & 0.132 \\
\hline Bandemia (No. of patients) & $15(55.6 \%)$ & $23(37.7 \%)$ & 0.119 \\
\hline $\mathrm{Hb}(\mathrm{g} / \mathrm{dL})$ & $10 \pm 2.4$ & $11 \pm 2.4$ & 0.056 \\
\hline Platelet $\left(\times 10^{3} / \mu \mathrm{L}\right)$ & $244.7 \pm 205.2$ & $264.5 \pm 145.0$ & 0.234 \\
\hline BUN (mg/dL) & $55.4 \pm 55.0$ & $37.9 \pm 41.9$ & 0.053 \\
\hline Creatinine $(\mathrm{mg} / \mathrm{dL})$ & $2.5 \pm 2.1$ & $2.2 \pm 2.8$ & 0.158 \\
\hline $\operatorname{ALT}(U / L)(n=27$ vs 60) & $68.5 \pm 110.4$ & $78.8 \pm 225.3$ & 0.287 \\
\hline $\mathrm{Na}(\mathrm{mEq} / \mathrm{L})$ & $132.3 \pm 6.5$ & $134.2 \pm 6.5$ & 0.132 \\
\hline $\mathrm{K}(\mathrm{mEq} / \mathrm{L})$ & $4.3 \pm 0.8$ & $4.1 \pm 0.7$ & 0.178 \\
\hline $\mathrm{CRP}(\mathrm{mg} / \mathrm{L})$ & $217.8 \pm 92.8$ & $184.8 \pm 116.9$ & 0.168 \\
\hline CK (U/L) ( $n=22$ vs 54) & $207.4 \pm 360.3$ & $314.3 \pm 1150.0$ & 0.439 \\
\hline $\begin{array}{l}\text { Initial blood glucose } \\
\text { (mg/dL) ( } \mathrm{n}=27 \text { vs 59) }\end{array}$ & $211.4 \pm 139.9$ & $170.0 \pm 115.9$ & 0.098 \\
\hline Primary:Secondary origin & $3: 24$ & 18:43 & 0.062 \\
\hline Mortality & $12(44.4 \%)$ & 10(16.4\%) & $0.005^{*}$ \\
\hline Length of hospital stay (days) & $37.6 \pm 32.8$ & $35.3 \pm 41.6$ & 0.906 \\
\hline
\end{tabular}

Fisher's Exact Test. * $\mathrm{P}<0.05$.

$\mathrm{ALT}$, alanine aminotransferase; $\mathrm{BUN}$, blood urea nitrogen; $\mathrm{CK}$, creatine kinase; CKD, chronic kidney disease; CRP, C-reactive protein; CT, computed tomography; $\mathrm{DM}$, diabetes mellitus; $\mathrm{Hb}$, hemoglobin; IPA, iliopsoas abscess; $\mathrm{K}$, potassium; $\mathrm{Na}$, sodium; SD, standard deviation; WBC, white blood cells.

$82.0 \%(50 / 61)$ in patients with non-gas-forming IPA. The distribution of pathogens in blood and pus cultures is summarized in Table 5 . The predominant etiological micro-organisms in the non-gas forming IPA group were gram-positive cocci (GPC); mainly Staphylococcus spp. The incidences of GPC and gram-negative bacilli (GNB) were equal in the gas-forming IPA group. The incidence of GNB in blood or pus cultures was higher in the gas-forming IPA group than in the non-gas forming IPA group $(51.9 \%$ versus $27.9 \%)(P<0.05)$. The same trend was found for the anaerobes (37.0\% versus $14.8 \%)$ $(P<0.05)$.

All patients in this study were initially treated with empirical antibiotics. Only 2 among the 13 gas-forming IPA patients initially undergoing PCD had a successful outcome (success rate $=15.4 \%$ ); 3 of the 11 IPA patients with failed initial PCD expired; and 8 of the 11 patients with failed initial PCD underwent salvage operation, of whom 5 survived. Seven of the 8 gas-forming IPA patients who underwent primary surgical intervention survived (success rate $=87.5 \%$ ). Six gas-forming IPA patients were treated with antibiotics alone without PCD or surgical intervention, only 1 of whom survived (success rate = $16.7 \%)$. In the non-gas forming IPA group, 23 of 61 patients initially underwent PCD; success was achieved in 17 patients (success rate $=73.9 \%$ ). The success rate of PCD was higher in the non-gas forming than in the gas-forming IPA group $(P<0.01)$ (Table 6$)$. Nineteen of 20 non-gas forming IPA patients undergoing primary operation survived. The survival rates of patients who received antibiotics alone in the gas-forming IPA and non-gas forming IPA groups were $16.7 \%(1 / 6)$ and $61.1 \%(11 / 18)$, respectively. The treatment approaches in the gas-forming IPA and non-gas forming IPA groups are delineated in Figure 1. We also conducted a Kaplan-Meier analysis to compare the survival rate within 60 days between the 2 groups. Log rank test showed a higher survival rate in the non-gas forming IPA group (77.9\%) than in the gas-forming group $(51.7 \%)(P<0.05)$ (Figure 2).

\section{Discussion}

Iliopsoas muscles are susceptible to infections from distant sites and contiguous structures because of their rich blood supply and overlying retroperitoneal lymphatic systems. IPA is often overlooked because of its insidious onset, and subsequent severe sepsis may be life-threatening. Although the exact incidence of IPA is unknown, more and more cases are being identified because of widespread application of CT in suspected IPA patients. Despite recent advances in diagnostic modalities, the mortality rate of IPA has not improved much $(6.7 \%$ in a study by Ricci in 1986 and 5\% in a study by Navarro in 2009) $[2,12]$. In our study, the mortality rate in IPA patients $(22 / 88,25 \%)$ was higher than that reported in a meta-analysis conducted by Lai et al. $(8 \%, 55 / 682$, between 1986 and 2011) [9]. However, because those patients were from various medical settings in different countries, the results of that meta-analysis cannot be used as the gold standard for clinical comparison, and 
Table 5 Distribution of pathogens between gas-forming IPA and non-gas forming IPA patients

\begin{tabular}{|c|c|c|c|c|}
\hline & \multicolumn{2}{|c|}{ Gas-forming $n=27$} & \multicolumn{2}{|c|}{ Non-gas forming $n=61$} \\
\hline & Blood & Pus & Blood & Pus \\
\hline GPC & $10(37.0 \%)$ & $12(44.4 \%)$ & $30(49.2 \%)$ & $28(45.9 \%)$ \\
\hline Staphylococcus spp. & 6 & 6 & 21 & 20 \\
\hline MRSA & 3 & 2 & 7 & 7 \\
\hline OSSA & 3 & 3 & 11 & 11 \\
\hline Coagulase-negative Staphylococcus & 0 & 1 & 3 & 2 \\
\hline Streptococcus spp. & 4 & 4 & 8 & 6 \\
\hline Enterococcus & 0 & 2 & 1 & 2 \\
\hline GNB & $9(33.3 \%)$ & $14(51.9 \%)$ & $6(9.8 \%)$ & $13(21.3 \%)$ \\
\hline Escherichia coli & 1 & 5 & 1 & 4 \\
\hline Klebsiella pneumoniae & 3 & 4 & 0 & 6 \\
\hline \multicolumn{5}{|l|}{ Others } \\
\hline Anaerobics & $3(11.1 \%)$ & $9(33.3 \%)$ & $4(6.6 \%)$ & $6(9.8 \%)$ \\
\hline Fungus & 0 & $1(3.7 \%)$ & $2(3.3 \%)$ & $3(4.9 \%)$ \\
\hline TB & 0 & 0 & 0 & $1(1.6 \%)$ \\
\hline Mixed flora & $3(11.1 \%)$ & 7 (25.9\%) & $8(13.1 \%)$ & $11(18.0 \%)$ \\
\hline MDR isolates & $2(7.4 \%)$ & $3(11.1 \%)$ & 0 & $3(4.9 \%)$ \\
\hline
\end{tabular}

GNB, Gram-negative bacilli; GPC, Gram-positive cocci; IPA, iliopsoas abscess; MDR, multiple-drug resistant; MRSA, methicillin-resistant Staphylococcus aureus; OSSA, oxacillin-sensitive Staphylococcus aureus.

further clarification is needed. Among the 88 patients in our study group, 24 (27.3\%) had already been hospitalized in other hospitals and were referred to us because of poor treatment response. The mortality rate among these patients was high $(7 / 24,29.2 \%)$. The remaining patients $(64 / 88,72.7 \%)$ were admitted via our emergency department (ED) with initial severe illness. In addition, severe sepsis was frequently observed in our total study group. The general poor health of the patients was probably the major cause of high mortality in the study group. In addition, at our hospital, more conservative treatment policies were adopted because of patients' older age, which itself might have contributed to a higher mortality rate. Other possible explanations of the high mortality rate include large number of gas-forming IPA cases and predominance of secondary IPA.
The presentations of IPA in our study varied, including fever, flank pain or back pain, abdominal pain, thigh lumps, altered consciousness, and even shock. The initial nonspecific symptoms; e.g., nausea, general weakness, intermittent mild fever, or low back soreness, might easily be ignored by patients. We attempted to evaluate how long the patients had been ill via a complete medical record review. Pre-presentation symptoms could not be evaluated definitively in 24 of 88 patients $(27.3 \%)$ who had stayed in other hospitals for periods of time. A higher mortality rate $(7 / 24,29.2 \%)$ was noted in this group. Additionally, the morphology of IPA pattern, according to the images in our hospital, might have been influenced by prior therapy. Sixty-four patients $(72.7 \%)$ in our study were directly referred from another ED because of severe illness or initially sought medical

Table 6 Success rate of different management pathways between gas-forming IPA and non-gas forming IPA patients

\begin{tabular}{|c|c|c|c|}
\hline & $\begin{array}{l}\text { Gas-forming IPA } \\
\qquad(n=27)\end{array}$ & $\begin{array}{l}\text { Non-gas forming IPA } \\
\qquad(\mathrm{n}=61)\end{array}$ & $P$ value \\
\hline Total number of initial treatments with PCD & $13(48.2 \%)$ & $23(37.7 \%)$ & 0.358 \\
\hline PCD success rate & $2 / 13(15.4 \%)$ & $17 / 23(73.9 \%)$ & $0.0007^{* *}$ \\
\hline Total number undergoing surgical debridement & $16(59.3 \%)$ & $25(41.0 \%)$ & 0.113 \\
\hline Primary operation success rate ${ }^{f}$ & $7 / 8(87.5 \%)$ & 19/20 (95.0\%) & 0.497 \\
\hline Salvage operation success rate (in patients with failed PCD) ${ }^{f}$ & $5 / 8(62.5 \%)$ & $4 / 5(80.0 \%)$ & 1.000 \\
\hline Antibiotics alone success rate ${ }^{f}$ & $1 / 6(16.7 \%)$ & $11 / 18(61.1 \%)$ & 0.155 \\
\hline
\end{tabular}

${ }^{{ }^{F} \text { Fisher's Exact Test. }{ }^{* *} \mathrm{P}<0.001 .}$ 


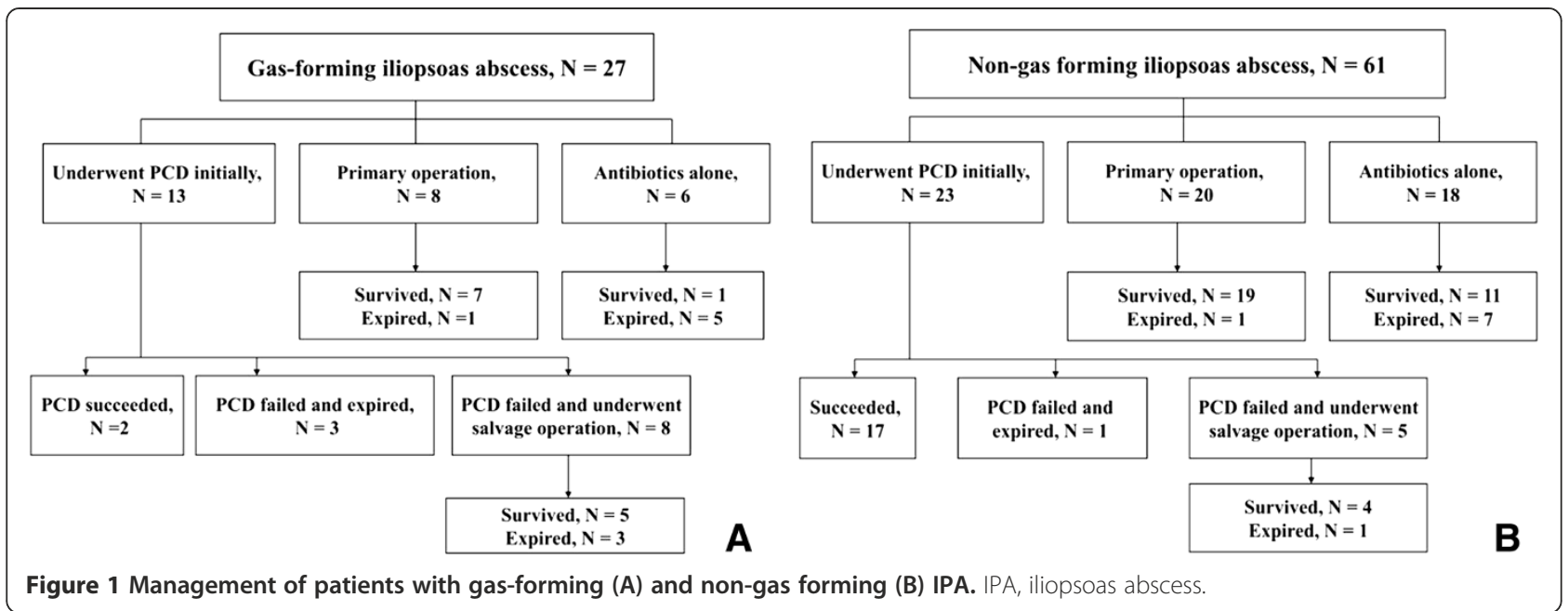

assistance in our hospital. The majority of patients in this group $(55 / 64,85.9 \%)$ had a long illness prior to presentation; 12.55 days on average. Among these 55 patients, 17 (30.9\%) had bilateral IPA and 49 (89.1\%) had multiloculated IPA. The remaining 9 patients (9/ $64,14.1 \%$ ) in this group could not be evaluated because medical records were unclear or because patients had deterioration of consciousness upon initial presentation and ultimately expired. In brief, the late presentation of most of our patients may have contributed to the high total mortality.

IPA is clinically classified into primary and secondary origins according to its initial infection site. In 1986, Ricci et al. described 286 cases of primary IPA, mainly young patients from developing countries [2]. There were 90 cases of secondary IPA, which were almost exclusively from developed countries, and the most common etiologies were Crohn's disease, followed by appendicitis and colon inflammation or cancer. Since then, more and more patients have been found to have secondary rather than primary IPA. In 2009, Navarro et al. reported 124 cases in a multi-center study, $78.2 \%$ with secondary IPA, and a single-center study by Carolyn et al. in 2001 demonstrated $80 \%$ of 61 cases having secondary IPA. The main sources of secondary IPA in the study by Navarro et al. were skeletal (50.5\%), followed by gastrointestinal tract (24\%) and genitourinary tract $(17.5 \%)$. Our study revealed a similar trend with predominance of secondary IPA (76.1\%) in which the most common infection origins were skeletal, followed by cardiovascular system and urinary tract. The higher prevalence of secondary IPA reported recently and in our study may be attributable to the widespread application of CT and MRI [13]. These imaging modalities can clearly delineate adjacent structures, especially the vertebrae and epidural space, and it is therefore likely that the infectious focus will be clarified. Furthermore, in our report, 11 patients of secondary IPA originated from cardiovascular system, including abdominal aortic aneurysm (AAA) post-stent implantation with subsequent infection, infected aortic aneurysm, and infective endocarditis, all of which have rarely been mentioned in the literature $[14,15]$.

Gas-formation is an important predictor of clinical outcome for patients with liver abscess and acute pyelonephritis $[16,17]$. Furthermore, in patients with acute pyelonephritis, the treatment policy depends mainly on whether the gas-forming (emphysematous) change is detected within the urinary tract. The major components of the gas in emphysematous pyelonephritis are nitrogen, hydrogen, carbon dioxide, and oxygen, and mixed acid fermentation has been proposed as the major mechanism of gas production because of the hydrogen content $[17,18]$. Other authors have indicated that rapid tissue catabolism complicated by impaired transport of end products around the infection site produces the gas [19]. Based on the clinical consensus that in acute pyelonephritis gas formation is the major criterion for further treatment decisions, we propose a new algorithm for determining treatment modality in IPA patients according to the presence of gas. In our study, GNB and anaerobic infections were more often discovered in patients with gas-forming IPA than in those with non-gas forming IPA. This phenomenon may explain the mechanism of gas formation and the development of a fulminant course of IPA.

Abscess drainage plus antibiotic treatment is essential for appropriate management of IPA patients. Options for drainage are surgery or PCD. Generally, IPA patients with concurrent intra-abdominal or retroperitoneal abnormalities such as ruptured appendicitis or ruptured infected aortic aneurysm require surgery to clean out or repair the infected foci. Surgical intervention may provide more effective drainage than PCD, especially in patients with multiloculated IPA [4]. IPA patients were often treated 


\section{Survival rate (60-day)}

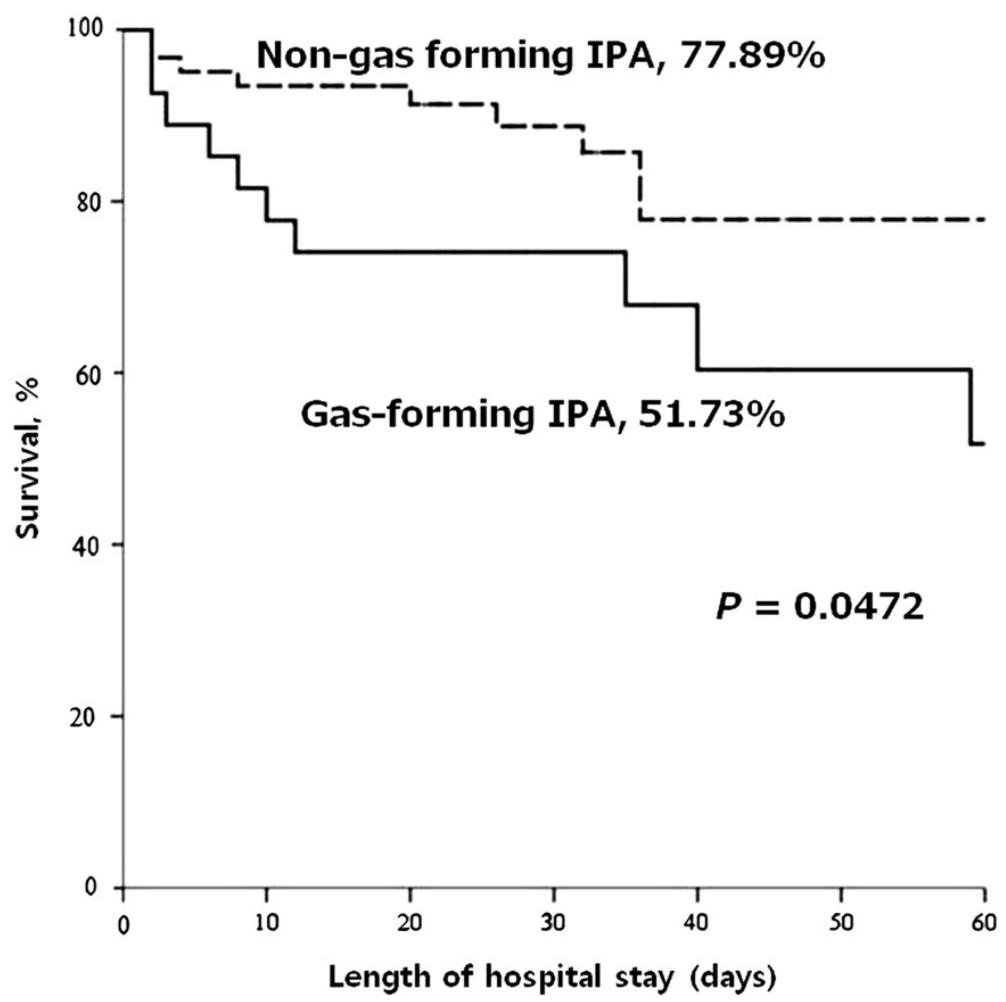

Figure 2 Kaplan-Meier analysis of cumulative survival rate during a 60-day hospital stay between patients with gas-forming and non-gas forming IPA. IPA, iliopsoas abscess.

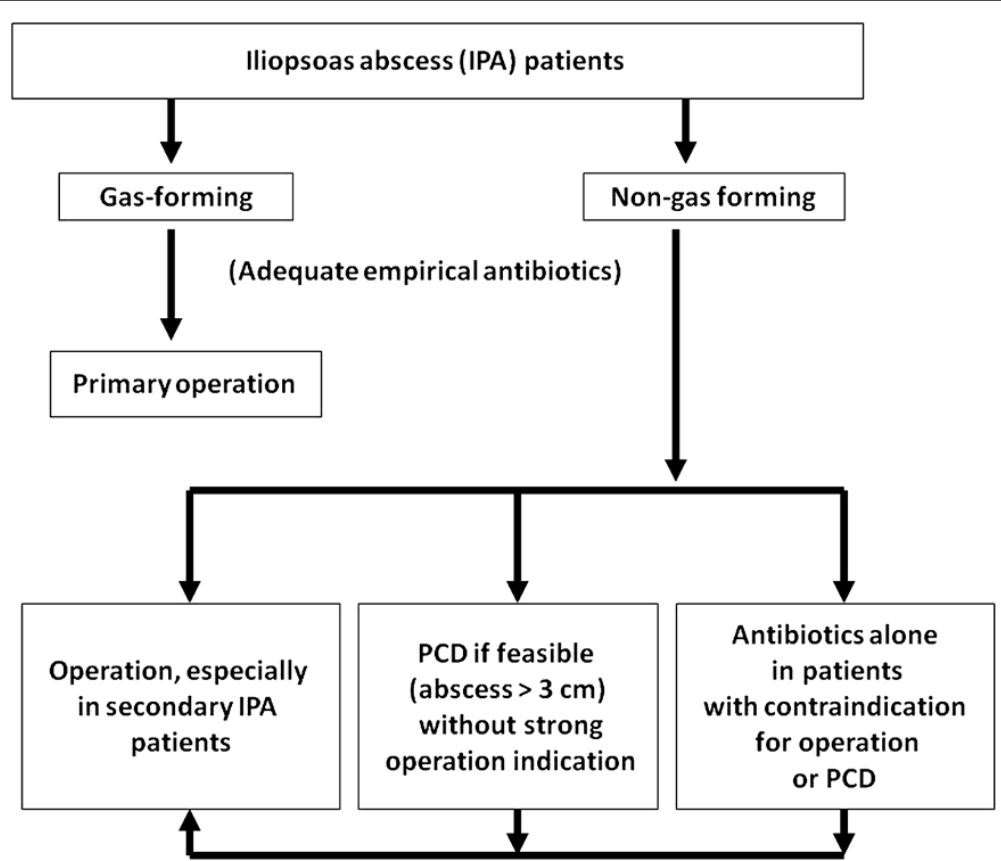

Figure 3 Suggested clinical management algorithm for IPA patients. IPA, iliopsoas abscess. 
with surgical debridement in the 1980s, when imaging techniques were still uncommon [2]. Afaq et al. also reported no mortality in 72 cases in Nepal in which all of the patients received surgical intervention as the first choice of treatment [20]. Recent studies have suggested that IPA can be successfully treated with antibiotics plus PCD [11,21], but not all of the cases in our study could be cured by PCD, especially when the IPA was gas-forming. If there are strong indications for primary operation, such as ruptured infected aortic aneurysm, ruptured appendicitis, or epidural abscess with spinal cord compression, surgical intervention should not be delayed. Furthermore, in our study, surgical intervention was preferred when gas-forming IPA was observed because of the higher failure rate of PCD. However, PCD plus appropriate antibiotics is adequate for treating patients with non-gas forming and solitary IPA. In addition, PCD remains an option for IPA patients who are not suitable for operation under general anesthesia. The suggested treatment algorithm based on the results of our study is delineated in Figure 3.

The major strength of this study is the use of a large number of IPA patients from a single center. Although previous multi-center study used larger sample sizes, many relevant variables could not be evaluated because of differences in record format across medical centers. However, there are several limitations to this study. First, there is no international consensus for IPA treatment; treatment policy depends on the treating physicians, whose training and clinical experience may play roles. Second, no matter which modality of treatment is chosen, the origin of infection may affect the clinical outcome, and this could not be evaluated in this study. Third, recurrence was not evaluated in this study. Thus, it is unknown whether repeated PCD or surgical intervention was performed. Fourth, the complexity of the disease varied in our sample.

\section{Conclusions}

Our findings suggest that classification of IPA patients into gas-forming IPA or non-gas forming IPA groups may help physicians make early and definitive decisions with regard to treatment choices. Our study showed that PCD treatment for patients with gas-forming IPA resulted in a high failure rate and increased intra-hospital mortality rate. We recommend early surgical intervention with appropriate antibiotic treatment for gas-forming IPA patients. PCD is adequate for the management of patients with solitary and non-gas forming IPA, especially for primary IPA.

\section{Competing interests}

The authors declare they have no competing interests.

\section{Authors' contributions}

M-SH, J-AH, and S-YH participated in study design. M-SH, S-CH, Y-YH, C-AT, and $Y-T T$ participated in data acquisition, analysis, and interpretation. Critical revision of the manuscript for important intellectual content: $\mathrm{M}-\mathrm{SH}, \mathrm{E}-\mathrm{WL}$, L-MW, and S-YH. All authors read and approved the final manuscript.

\section{Acknowledgements}

We thank the Biostatistics Task Force of Taichung Veterans General Hospital, Taichung, Taiwan, ROC, for their assistance and advice in statistical analysis.

\section{Author details}

'Department of Emergency Medicine, Taichung Veterans General Hospital, Taichung, Taiwan. ${ }^{2}$ School of Medicine, Chung Shan Medical University, Taichung, Taiwan. ${ }^{3}$ Institute of Medicine, Chung Shan Medical University, Taichung, Taiwan. ${ }^{4}$ Kaohsiung Municipal Kai-Syuan Psychiatric Hospital, Kaohsiung, Taiwan. ${ }^{5}$ Division of Infectious Disease, Department of Internal Medicine, Taichung Veterans General Hospital, Taichung, Taiwan. ${ }^{6}$ Department of Radiology, Taichung Veterans General Hospital, Taichung, Taiwan. ${ }^{7}$ Department of Health Service Administration, China Medical University, Taichung, Taiwan. ${ }^{8}$ School of Medicine, National Yang-Ming University, Taipei, Taiwan. ${ }^{9}$ National Defense Medical Center, Taipei, Taiwan. ${ }^{10}$ Department of Nursing, College of Health, National Taichung University of Science and Technology, No. 1650 Taiwan Boulevard Sect. 4, Taichung 40705, Taiwan

Received: 6 July 2013 Accepted: 27 November 2013 Published: 9 December 2013

\section{References}

1. Mallick IH, Thoufeeq MH, Rajendran TP: lliopsoas abscesses. Postgrad Med J 2004, 80(946):459-462.

2. Ricci MA, Rose FB, Meyer KK: Pyogenic psoas abscess: worldwide variations in etiology. World J Surg 1986, 10(5):834-843.

3. Buttaro M, Gonzalez Della Valle A, Piccaluga F: Psoas abscess associated with infected total hip arthroplasty. J Arthroplasty 2002, 17(2):230-234.

4. Santaella RO, Fishman EK, Lipsett PA: Primary vs secondary iliopsoas abscess: presentation, microbiology, and treatment. Arch Surg 1995, 130(12):1309-1313.

5. Chern CH, Hu SC, Kao WF, Tsai J, Yen D, Lee CH: Psoas abscess: making an early diagnosis in the ED. Am J Emerg Med 1997, 15(1):83-88.

6. Kao PF, Tsui KH, Leu HS, Tsai MF, Tzen KY: Diagnosis and treatment of pyogenic psoas abscess in diabetic patients: usefulness of computed tomography and gallium-67 scanning. Urology 2001, 57(2):246-251.

7. Tonolini M, Campari A, Bianco R: Common and unusual diseases involving the iliopsoas muscle compartment: spectrum of cross-sectional imaging findings. Abdom Imaging 2012, 37(1):118-139.

8. Cronin CG, Lohan DG, Meehan CP, Delappe E, McLoughlin R, O'Sullivan GJ, McCarthy P: Anatomy, pathology, imaging and intervention of the iliopsoas muscle revisited. Emergency radiology 2008, 15(5):295-310.

9. Lai YC, Lin PC, Wang WS, Lai Jl: An update on Psoas muscle abscess: an 8-year experience and review of literature. Int J Gerontol 2011, 5(2):75.

10. Yamagami T, Terayama K, Yoshimatsu R, Matsumoto T, Miura H, Nishimura T: Percutaneous drainage of psoas abscess under real-time computed tomography fluoroscopic guidance. Skeletal Radio/ 2009, 38(3):275-280.

11. Yacoub WN, Sohn HJ, Chan S, Petrosyan M, Vermaire HM, Kelso RL, Towfigh S, Mason RJ: Psoas abscess rarely requires surgical intervention. Am J Surg 2008, 196(2):223-227.

12. Navarro Lopez V, Ramos JM, Meseguer V, Perez Arellano JL, Serrano R, Garcia Ordonez MA, Peralta G, Boix V, Pardo J, Conde A, et al: Microbiology and outcome of iliopsoas abscess in 124 patients. Medicine (Baltimore) 2009, 88(2):120-130.

13. Gruenwald I, Abrahamson J, Cohen O: Psoas abscess: case report and review of the literature. J Urol 1992, 147(6):1624-1626.

14. Chan JF, Hwang GY, Lamb S, Chan GS, So JC, Leung SS, To KK, Li IW, Cheng VC, Yuen KY: Pneumococcal native aortic valve endocarditis with mycotic abdominal aortic aneurysm, paraspinal and iliopsoas abscesses and pneumonia revealing a multiple myeloma. J Med Microbio/ 2011, 60(Pt 6):851-855.

15. Hsu RB, Lin FY: Psoas abscess in patients with an infected aortic aneurysm. J Vasc Surg 2007, 46(2):230-235. 
16. Chen SC, Tsai SJ, Chen CH, Huang CC, Lin DB, Wang PH, Chen CC, Lee MC Predictors of mortality in patients with pyogenic liver abscess. Neth $J$ Med 2008, 66(5):196-203

17. Huang JJ, Tseng CC: Emphysematous pyelonephritis: clinicoradiological classification, management, prognosis, and pathogenesis. Arch Intern Med 2000, 160(6):797-805.

18. Huang JJ, Chen KW, Ruaan MK: Mixed acid fermentation of glucose as a mechanism of emphysematous urinary tract infection. J Urol 1991, 146(1):148-151.

19. Yang WH, Shen NC: Gas-forming infection of the urinary tract: an investigation of fermentation as a mechanism. J Urol 1990, 143(5):960-964.

20. Afaq A, Jain BK, Dargan P, Bhattacharya SK, Rauniyar RK, Kukreti R: Surgical drainage of primary iliopsoas abscess-safe and cost-effective treatment. Trop Doct 2002, 32(3):133-135.

21. Cantasdemir M, Kara B, Cebi D, Selcuk ND, Numan F: Computed tomographyguided percutaneous catheter drainage of primary and secondary iliopsoas abscesses. Clin Radiol 2003, 58(10):811-815.

doi:10.1186/1471-2334-13-578

Cite this article as: Hsieh et al:: Features and treatment modality of iliopsoas abscess and its outcome: a 6-year hospital-based study. BMC Infectious Diseases 2013 13:578.

\section{Submit your next manuscript to BioMed Central and take full advantage of:}

- Convenient online submission

- Thorough peer review

- No space constraints or color figure charges

- Immediate publication on acceptance

- Inclusion in PubMed, CAS, Scopus and Google Scholar

- Research which is freely available for redistribution 